\title{
Organiser la rencontre entre chercheurs et enseignants : finalités, moyens, dispositifs
}

\section{Vincent Grosstephan}

\section{(2) OpenEdition}

1 Journals

\section{Édition électronique}

URL : http://journals.openedition.org/trema/2935

DOI : 10.4000/trema.2935

ISSN : 2107-0997

\section{Éditeur}

Faculté d'Éducation de l'université de Montpellier

\section{Édition imprimée}

Date de publication : 1 juin 2013

Pagination : $36-49$

ISBN : 1167-315X

ISSN : 1167-315X

\section{Référence électronique}

Vincent Grosstephan, «Organiser la rencontre entre chercheurs et enseignants : finalités, moyens, dispositifs », Tréma [En ligne], 39 | 2013, mis en ligne le 01 juin 2015, consulté le 01 mai 2019. URL http://journals.openedition.org/trema/2935; DOI : 10.4000/trema.2935

Ce document a été généré automatiquement le 1 mai 2019.

Trema 


\title{
Organiser la rencontre entre chercheurs et enseignants : finalités, moyens, dispositifs
}

\author{
Vincent Grosstephan
}

1 Cet article a pour but de s'interroger sur la nature des rapports entre recherche et enseignement qui est au cœur de nombreux débats actuels sur la professionnalisation des enseignants. Ainsi, dans le cadre du processus de réforme de la formation des maîtres dit processus de «mastérisation", la place des recherches en éducation est fortement interrogée. Les finalités épistémique (production de savoirs), formative et pragmatique (transformation de la réalité) de ces recherches sont ainsi discutées à la fois dans leurs spécificités et dans leur articulation. Nombreux sont ceux qui s'interrogent sur la possibilité et la

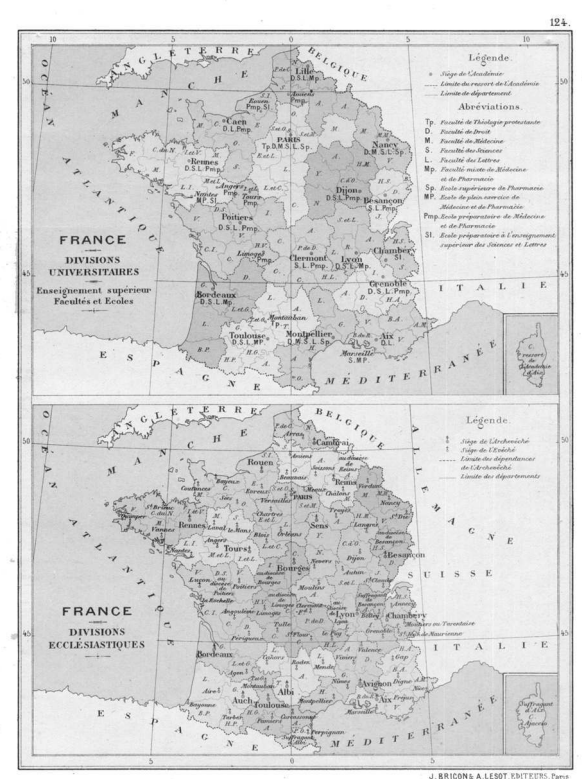
légitimité de jouer sur ces deux ou trois tableaux dans le cadre de dispositifs associant étroitement chercheurs et enseignants. Certes cette interrogation n'est pas nouvelle et a déjà fait l'objet de nombreux débats notamment à propos des recherchesaction. Pour autant, il nous semble que ces questions ressurgissent aujourd'hui sous un éclairage nouveau, en raison d'une part des évolutions de la formation d'adultes (Barbier, 2001) et d'autre part de changements paradigmatiques importants dans les recherches en éducation et formation (Schurmans, 2008). Nous allons ainsi, dans un premier temps, tenter de caractériser le mouvement de fond général relatif à la formation des adultes avant de proposer une analyse critique de différents types de dispositifs associant 
explicitement chercheurs et enseignants. Nous avons ainsi identifié neuf modalités dont certaines peuvent être considérées comme extrêmement proches du point de vue de leurs caractéristiques et de leur mise en œuvre mais que nous avons néanmoins souhaité distinguer pour des raisons tantôt théoriques, tantôt méthodologiques. Ces modalités concernent des recherches sur, avec ou pour les enseignants, certaines combinant deux ou trois de ces orientations. Ces neuf dispositifs recouvrent ainsi peu ou prou l'ensemble des modalités d'association chercheurs-praticiens que nous avons pu identifier dans le champ de l'éducation et de la formation.

\section{Les évolutions de la formation d'adultes}

2 L'étude des rapports complexes entre recherche et enseignement au sein de dispositifs associant des chercheurs et des enseignants ne peut être dissociée d'une interrogation sur un mouvement de fond plus général relatif à la formation des adultes. Barbier (2001) voit dans ce mouvement une "recomposition " à la fois de la fonction de la formation mais aussi de ses modalités et dispositifs, tout cela sur fond de "crise du monde de la formation " (baisse sensible des moyens privés et publics affectés à la formation d'adultes; nouveaux dispositifs intégrant travail, communication et développement de compétences ; passage d'une culture de la formation à une culture de la professionnalisation; modification en nombre, statut et activité du groupe professionnel des formateurs). Ces évolutions ont probablement pour origine, selon Barbier (2006, p. 71) «les mutations économiques et sociales tendant à privilégier le pilotage de la production par la demande et la recherche de la productivité à partir du facteur humain ». Il identifie ainsi un certain nombre d'évolutions de la fonction formation :

- tout d'abord une finalisation plus forte de la formation par rapport aux situations suscitant son recours : il s'agit là d'une volonté marquée de passer d'une logique de l'offre générale à une logique de la demande locale : stages d'équipes pédagogiques, stages d'établissements, stages de bassin de formation etc. ;

- apparition de nouvelles logiques d'articulation entre formation, recherche et action. Barbier y voit notamment l'apparition d'un refus de situations de formation aux caractéristiques d'espace protégé et autonome. Ce refus tendrait ainsi à promouvoir de nouvelles modalités de formation, plus collaboratives, plus situées, prenant plus fortement en compte les pratiques réelles dans leur singularité, leur contingence, leurs enjeux, leurs charges de signification. Autant d'éléments à prendre en compte qui exigent de la part des formateurs des compétences nouvelles, notamment dans la forte dimension clinique de ces approches qui sont le plus souvent l'apanage de chercheurs en analyse du travail, en anthropologie cognitive, en didactique professionnelle etc. ;

- néanmoins, ces volontés nouvelles de pilotage de la formation par la demande, d'articulation plus étroite entre travail et formation, de redéfinition des rôles, missions et statuts des intervenants en formation peuvent, à certains moments, heurter la culture dominante des responsables chargés de la conception et de la gestion de ces dispositifs : cette culture dominante à l'éducation nationale, dans les rectorats et les inspections académiques reste celle de l'enseignement ce qui aboutit parfois à des difficultés à faire accepter aux responsables académiques de formation notamment la mise en place d'actions s'inscrivant clairement dans un espace de professionnalisation voire même de formation ; 
- enfin, Barbier voit dans ces évolutions de la formation d'adultes une pression à l'autonomisation des acteurs et des parcours aux niveaux individuel et collectif, induisant par là-même des enjeux de flexibilité identitaire.

Ces évolutions mises en évidence par Barbier nous renvoient à trois questions qui nous semblent fondamentales et qui vont organiser l'analyse critique des différents types de dispositifs de rencontre chercheurs-enseignants étudiés.

- La première concerne les finalités de la rencontre entre enseignants-chercheurs et enseignants dans ces nouveaux espaces et dispositifs. Nous considérons que ces finalités se situent, de façon variable selon le type de dispositif de rencontre envisagé, dans un espace délimité par trois pôles. La recherche, la formation et l'action ;

- La seconde question concerne les arrières plans épistémologiques de ces dispositifs. En privilégiant des approches situées et en accordant une importance forte au point de vue subjectif des acteurs, ces dispositifs renouvellent les paradigmes de recherche dominants et les manières d'appréhender les situations d'enseignement/apprentissage scolaires. Nous passons ainsi d'un objectivisme strict à la construction culturelle de significations, d'une approche explicative à une approche compréhensive. Ceci nous amène à questionner le point de vue mobilisé dans ces dispositifs : le point de vue extrinsèque du chercheur ou de l'analyste et/ou le point de vue intrinsèque de l'enseignant (part conscientisable de son activité). Ces orientations épistémologiques convergentes renvoient cependant à une diversité importante de cadres théoriques dont nous tenterons de rendre compte succinctement.

- De nombreux travaux s'inspirant des démarches collaboratives mettent en évidence une situation « en tension » pour l'enseignant-chercheur engagé dans des dispositifs de ce type : nous nous posons dès lors la question de savoir quelles peuvent être la posture, l'activité et l'engagement de cet enseignant-chercheur : chercheur? Formateur ? Co-constructeur? Nous interrogerons également la possibilité pour cet enseignant-chercheur d'assumer simultanément ces différents rôles au sein d'un dispositif dont il est un des principaux acteurs. De façon équivalente à la question précédente, nous nous questionnerons sur la posture et l'activité des enseignants dans ces dispositifs : formés ? Sujets ? Co-constructeur? Et la possibilité d'assumer simultanément ces différents rôles.

4 Nous avons pris l'option de présenter les différentes modalités de rencontre entre enseignants-chercheurs et enseignants identifiées, à travers le prisme des trois questions posées ci-dessus.

\section{Les finalités des rencontres chercheurs - enseignants}

5 Le schéma $n^{\circ} 1$ montre de façon générale que si les trois pôles de la recherche, de la formation et de l'action structurent les finalités et les caractéristiques de ces dispositifs c'est, à l'exception des recherches descriptives et compréhensives sur l'enseignement, dans l'articulation de deux, voire trois d'entre elles que se positionnent réellement ces dispositifs de rencontre (Grosstephan, 2010). 
Schéma $n^{\circ} 1$ : espace des finalités des dispositifs de rapprochement entre recherche et enseignement

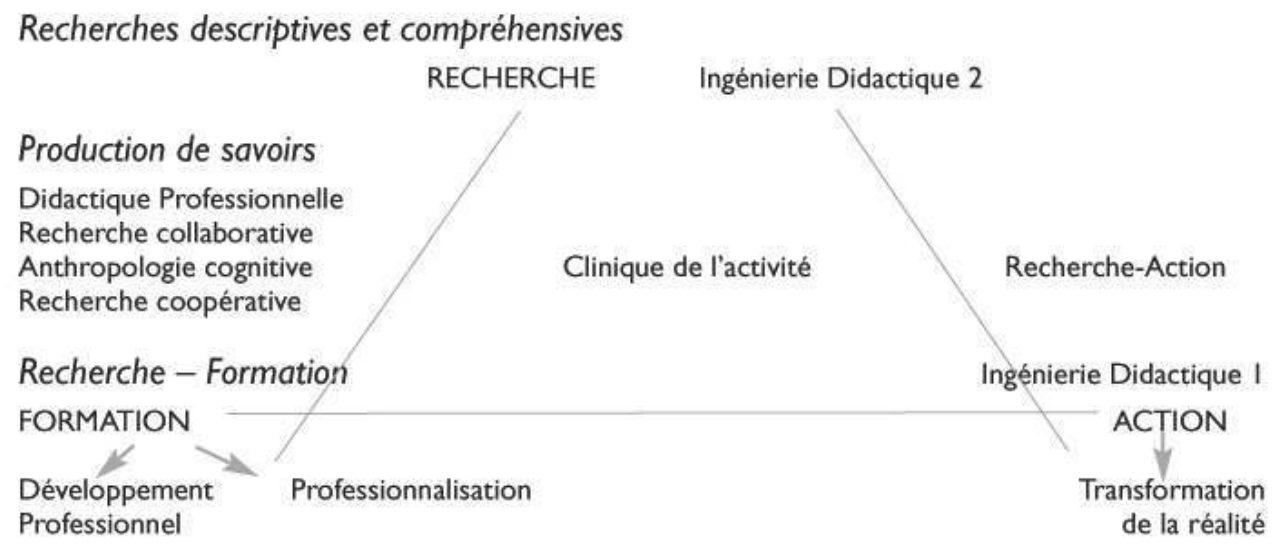

\section{1. Connaître la réalité sociale et participer à sa transformation : le point de vue de la recherche-action et de l'ingénierie didactique}

6 L'idée consistant à penser que pour mieux connaître une réalité il faut participer à sa transformation renvoie à certaines constantes qui traversent l'histoire de la rechercheaction et des dispositifs de même nature: la réponse à des besoins portés par les participants dans leur milieu, l'implication des acteurs, le processus de changement des pratiques et l'action concrète sur le terrain... Il semble bien in fine que ce qui fonde la spécificité de cette démarche c'est bien sa dimension pragmatique dans la mesure où la recherche est utilisée pour résoudre un problème particulier qui concerne l'action des praticiens, recherche dans laquelle ces praticiens sont fortement impliqués. En ce sens, et contrairement aux ambitions affichées, l'essentiel des recherches-action semblent plutôt validées par leur pouvoir de transformation d'une réalité que par la production de savoirs qui a tendance à rester le parent pauvre de ces démarches. C'est sans doute une des raisons qui justifie son succès en tant qu'outil d'action mais aussi les critiques en matière de recherche. Parmi ces critiques les deux principales sont celles relatives au manque de rigueur dans de nombreux travaux marqués pas un flou conceptuel et où l'enthousiasme de l'échange et du débat se substitue à l'exigence conceptuelle et méthodologique et celle relative à la croyance naïve que toute la science pourrait naitre de la recherche-action. " Il n'en est rien car certaines investigations exigent un niveau élevé de spécialisation et de sophistication des méthodes et des techniques, et un engagement total du chercheur, ce qui est impossible au praticien » (De Landsheere, 1992, p. 626 - 627).

7 Dans une perspective différente, l'ingénierie didactique, dont on peut situer l'émergence en didactique des mathématiques au début des années 1980, propose deux orientations. La première ${ }^{1}$ relève d'une perspective de transformation du système que l'on étudie : il s'agit « d'affirmer la possibilité d'une action rationnelle sur le système basée sur des connaissances préétablies» (ibid., p. 285). Cette visée pragmatique, que Brousseau (1986) définit comme la proposition d'éléments de phénoménotechnique utiles à l'enseignement, nous semble relever d'une démarche de rationalité technique dans la mesure où l'enseignant n'est considéré que comme un élément, une variable de la situation expérimentale. La seconde ${ }^{2}$ relève d'une perspective "fondamentale » pour qui l'ingénierie constitue un prétexte pour étudier le système didactique. Les méthodologies utilisées permettent, en plongeant 
le chercheur dans la complexité du système qu'il se donne pour objectif d'étudier, de mettre en évidence un certain nombre de phénomènes didactiques que des méthodologies externes (questionnaires, entretiens, tests...) ne permettaient pas de repérer et d'analyser.

\section{2. Une perspective résolument formative : la recherche-formation}

Fort des critiques adressées à la recherche-action Marcel (1999) propose une autre voie dans laquelle la recherche et la formation inter-agiraient en s'enrichissant, mais sans toutefois abandonner leur spécificité. Il parle dès lors de dispositif de rechercheformation qui pour lui ne peut et ne doit constituer un dispositif d'action au sens que lui donnait la recherche-action. La demande sociale qui est exprimée vis-à-vis de la recherche en éducation ne doit pas déboucher sur des actions de formation en contradiction avec les orientations de recherche développées sur les pratiques enseignantes (tant $\mathrm{du}$ point de vue des référents théoriques que des résultats empiriques). Or ces orientations sont avant tout descriptives et compréhensives et rejettent toute idée de prescription aux enseignants. L'auteur insiste sur le fait qu'en menant en collaboration une recherche, les partenaires ne poursuivent que des objectifs de formation. En ce sens, il ne s'agit pas de tenter à tout prix de tenir les deux bouts : produire des connaissances selon les critères de scientificité établis d'un côté, favoriser l'apprentissage/développement des participants à la recherche-formation de l'autre. La finalité est exprimée de la façon suivante : "L'objectif est à la fois précis et circonscrit, il ne s'agira pas de prétendre fournir une méthode prête à appliquer ni une solution clé en main, mais simplement de mettre au service des formés nos compétences de chercheur pour leur permettre de construire leur solution " (Marcel, 1999, p. 92). Cette démarche se caractérise donc très explicitement par une visée de formation des praticiens engagés dans le dispositif.

\section{3. Concilier des objectifs de production de savoirs scientifiques et des objectifs de formation}

9 La didactique professionnelle, les recherches menées dans le cadre du paradigme de l'anthropologie cognitive, les recherches collaboratives et coopératives constituent des dispositifs visant explicitement un double objectif de recherche et de formation. Ils sont fondés notamment sur l'idée que des projets de recherche dans la communauté de pratique (Wenger, 1998), impliquant les acteurs du milieu eux-mêmes, aidés par des chercheurs, constituent une modalité favorable au développement professionnel de ses membres. En didactique professionnelle et pour les recherches menées dans le cadre du paradigme de l'anthropologie cognitive l'objectif de formation se réalise de deux façons. Tout d'abord, grâce à la mobilisation par les acteurs d'une activité réflexive. En effet, par le dialogue instauré entre les chercheurs et les professionnels, ces derniers ont accès aux propositions des chercheurs. Ainsi, la confrontation des acteurs, dans la recherche compréhensive, au matériau empirique les concernant: enregistrements audio-vidéos, verbatim des entretiens... et aux descriptions, analyses et interprétations produites sur leur activité par le chercheur permettrait d'induire une activité et un enrichissement réflexif des enseignants impliqués dans la recherche. Les prises de conscience associées aux descriptions, analyses et interprétations des chercheurs peuvent permettre d'entrevoir de nouveaux possibles pour les professionnels concernés sur la base 
d'activités cognitives de généralisation ou de réorganisations conceptuelles à l'instar de ce qu'évoquent les travaux en didactique professionnelle lorsqu'ils traitent de développement professionnel. Cette tendance confirme l'analyse de Barbier relativement aux articulations de plus en plus fortes entre recherche, formation et action. En se rapprochant de l'action, la recherche semble offrir de nouvelles occasions de formation. Ce constat amène une remarque : si, dans la plupart des cas, les effets induits par la plus forte implication des acteurs au processus de recherche ne faisaient pas partie des intentions prioritaires des chercheurs, nombreux sont ceux qui aujourd'hui revendiquent cette visée dans leurs dispositifs. Certes, la demande d'aide qui est adressée à la recherche par les enseignants (et les professionnels en général) n'est pas nouvelle mais elle permet d'être éclairée, à la lumière de ces constats, d'un jour nouveau. Ainsi, la didactique professionnelle, les programmes de recherche empiriques et technologiques du courant d'ergonomie et d'anthropologie cognitive, les recherches collaboratives, les dispositifs d'ingénierie didactique, les recherches en clinique de l'activité mettent tous en avant les perspectives transformatives ouvertes par leurs travaux au sein desquels les méthodologies utilisées jouent un rôle essentiel. Ce rôle des méthodologies utilisées constitue un élément remarquable que l'on peut tirer de l'analyse de ces dispositifs. L'objectif de formation se concrétise ensuite dans l'association à la recherche compréhensive d'un programme technologique ${ }^{3}$ ou par l'orientation vers une « ingénierie des compétences", s'appuyant sur la notion de «situations potentielles de développement » (Mayen, 1999). Il s'agit ici de partir de l'idée que certaines situations de travail (réelles ou simulées) sont porteuses des conditions du développement et qu'une ingénierie des compétences doit se donner pour objectif de concevoir ou s'appuyer sur des situations de ce type à partir d'une prise en compte des évolutions du travail et de la question du développement dans le travail.

10 À l'instar de la didactique professionnelle et des autres dispositifs évoqués plus haut, le courant de recherche développé au sein de l'équipe de clinique de l'activité du CNAM, sous l'impulsion de Clot, s'inscrit dans une tradition de recherche en analyse du travail qui se caractérise par une double inscription dans la communauté scientifique et dans le monde professionnel. Néanmoins, la clinique de l'activité semble aller encore plus loin dans cette volonté de concilier des finalités différentes en essayant de jouer sur les trois tableaux. On peut qualifier leurs visées de pragmatiques dans la mesure où les recherches menées se font en réponse à des demandes de professionnels qui souhaitent qu'on les aide à élargir leur rayon d'action, leur pouvoir d'agir sur leur milieu et sur eux-mêmes (Clot, 1999 ; Clot et Faïta, 2000). Ces interventions en clinique de l'activité ne constituent jamais qu'un simple projet de connaissance ou de recherche. L'investigation scientifique est conçue ici comme un instrument (Rabardel, 1995) dont les professionnels peuvent disposer. Néanmoins, au-delà des conséquences de l'intervention sur l'organisation du travail et sur les actions des professionnels, la visée transformative est bien plus ambitieuse et se propose de seconder les collectifs de travail dans leurs efforts pour reprendre un développement contrarié et mis en souffrance, ce qui suppose d'aller audelà d'objectifs strictement pragmatiques. La visée de développement professionnel et personnel est ici au cœur du projet de la clinique de l'activité. Enfin, la recherche en clinique de l'activité peut être considérée comme une recherche fondamentale de terrain portant sur les invariants du développement du pouvoir d'agir au travail et ses empêchements. 


\section{Des options épistémologiques convergentes - des cadres théoriques et méthodologiques divers}

11 Deux grandes options épistémologiques caractérisent ces dispositifs : ils s'inscrivent tous dans des approches dites «situées" (malgré quelques critiques pointues formulées par Béguin et Clot, 2004) et accordent une grande importance au point de vue subjectif de l'acteur.

\section{1. Des approches situées}

12 Les approches situées renvoient à un renouvellement des idées sur la conception de l'action et de la cognition. Ce renouvellement consiste pour l'essentiel en un déplacement de l'analyse, du sujet vers des objets théoriques singuliers et considérés comme pertinents: les objets matériels, le contexte, les interactions sociales... Il s'agit de proposer une alternative à la psychologie cognitive computationnelle en insistant sur des analyses en situations réelles, en limitant le rôle fonctionnel des plans, en remettant en cause la manipulation formelle de représentations symboliques notamment. On trouve dans ces approches un certain nombre de fondements théoriques récurrents « produit de la rencontre entre la psychologie cognitive et le riche héritage historique du constructivisme social »(Grison, 2004, p. 27). On y trouve ainsi des références nombreuses à Vygotsky, aux courants interactionnistes (école de Chicago, éthnométhodologie, pragmatique du langage...), à la phénoménologie, à la microsociologie allemande... Mais, au-delà de ces convergences et récurrences, les cadres théoriques mobilisés marquent des différences qui sont au cœur des débats entre leurs auteurs.

13 La recherche dans la cadre du programme empirique en anthropologie et en ergonomie cognitive trouve son ancrage théorique au sein du cadre "sémiologique » développé par Theureau (2004, 2006). L'activité humaine est prise ici comme objet d'étude et de conception et renvoie à deux postulats théoriques principaux. Le premier est le postulat d'énaction (Varela, 1989) qui traduit l'idée que chaque individu est un acteur structurellement couplé avec son environnement. Ainsi, l'action est toujours une réalisation contextualisée, située, et porte l'empreinte du contexte physique et social dans lequel elle s'inscrit. On peut dès lors parler de co-détermination de l'action et de la situation. Le second postulat théorique est celui de conscience pré réflexive. Tout acteur est toujours engagé dans une activité de construction d'une signification personnelle de la situation. On peut considérer qu'il existe un couplage fort entre un processus d'attribution de sens et l'accomplissement même de l'action dont il émerge. La conséquence, au regard de notre questionnement, est ainsi évidente et essentielle : ce programme de recherche installe de fait le primat du point de vue de l'acteur, privilégiant une perspective intrinsèque et reléguant le point de vue extrinsèque du chercheur au second plan.

14 Les recherches collaboratives ont la particularité de se fonder sur l'idée que faire de la recherche «avec les praticiens » apportera des connaissances plus fines et pertinentes sur les pratiques que faire de la recherche «sur les praticiens ». Ce postulat repose sur la reconnaissance, notamment à l'appui des travaux de Schön $(1994,1996)$ sur l'épistémologie de l'agir professionnel, du savoir d'expérience de l'enseignant. Cette reconnaissance justifie dès lors la validité de la contribution de son point de vue d'acteur 
compétent à la production de connaissances sur les pratiques professionnelles. Cette position en cela est fortement imprégnée de socio-contructivisme dans la mesure où le savoir produit relève d'une co-construction entre le chercheur et le (ou les) praticien(s) tout en affirmant le primat aux savoirs d'expérience des acteurs et à leur position d'acteur social compétent. Certaines critiques très fortes adressées aux courants interactionnistes, tout particulièrement à l'ethnométhodologie à laquelle font fortement référence les promoteurs des recherches collaboratives, considèrent qu'un des dangers d'une posture constructiviste radicale est que le chercheur, restant si proche du discours des acteurs et adhérant totalement à leurs présupposés et à leur vision du monde, se borne à retranscrire, de façon seulement plus claire et plus élaborée, et en le reprenant à son compte, ce que les acteurs disent eux-mêmes. Or, les acteurs ne font pas toujours ce qu'ils disent, et ne disent pas toujours ce qu'ils font, soit parce qu'ils méconnaissent les déterminants sociaux de leurs actions, soit parce qu'ils les considèrent comme inavouables, sans que ce constat puisse être assimilé à un procès d'intention.

Dugal (2003) qui présente son travail comme une variante de la recherche collaborative adresse pourtant à cette dernière une critique à ce qu'il dit relever d'une radicalité socioconstructiviste. Il propose une perspective légèrement différente qu'il nomme recherche coopérative, à propos d'une recherche portant le conseil pédagogique en Éducation Physique et Sportive : «À la différence de la recherche collaborative, notre démarche de recherche coopérative, conformément aux négociations de départ dans l'équipe de recherche, s'appuie à la fois sur des entrées par la pratique et des entrées par la théorie ». Il s'agit ici de se différencier de la posture des recherches collaboratives qui selon lui privilégie le seul point de vue de l'acteur.

En s'intéressant au travail réel et aux conditions de développement de l'activité, la didactique professionnelle et la clinique de l'activité sont elles aussi amenées à rapatrier le sujet dans la situation (Béguin et Clot, 2004). En ce sens elles rompent avec la tradition cognitiviste computationnelle et peuvent, en première analyse, être classées dans les courants dits «situés ». Ces deux orientations ont en commun néanmoins de tenter de dépasser l'opposition entre une psychologie de l'individu qui s'intéresse au développement endogène du sujet et une psychologie des situations qui s'intéresse au développement exogène de la situation. C'est à travers l'activité « que se réalise le rapport entre donné et créé, fonctionnement et développement » (ibid., p. 45). Certes, un débat sur la question de la nature des invariants (du fonctionnement ou du développement) oppose Pastré et Clot, mais ne remet pas fondamentalement en cause les convergences fortes entre ces deux perspectives de recherche et de formation qui, tout en rapatriant le sujet dans la situation, tentent également de le réincarner dans une perspective historicoculturelle. En cela, ils proposent une alternative aux approches situées radicales.

\section{2. L'importance accordée au point de vue de l'acteur}


Schéma $n^{\circ} 2$ : importance accordée par les dispositifs de rencontre chercheurs-praticiens au point de vue subjectif de l'acteur.

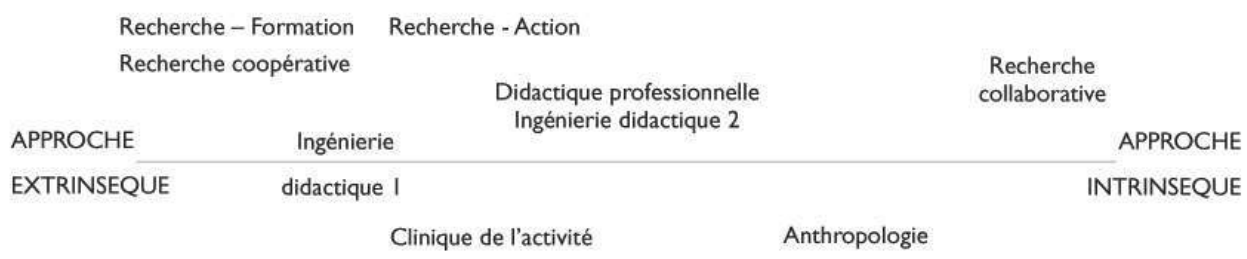

$$
\begin{aligned}
& \text { Recherches descriptives } \\
& \text { et compréhensives }
\end{aligned}
$$

cognitive

À l'exception du dispositif d'ingénierie didactique 1 tous les dispositifs associant chercheurs et enseignants accordent une grande importance au point de vue des acteurs. Cette tendance se justifie pour une raison principale : l'observation extérieure ne permet pas d'accéder aux significations construites par les acteurs en contexte. En effet, il est aujourd'hui postulé que toute action humaine, a fortiori une action de travail, est une activité cognitive de production de significations.

Pour Mouchet (2003, p. 91) il parait « indispensable dans l'analyse de l'activité de valoriser la subjectivité des acteurs, considérée non pas en tant qu'obstacle à l'objectivité et à la production de connaissances, mais au contraire comme validation intrinsèque et source formidable de documentation des zones d'ombre, ainsi que comme nécessité pour une intervention pertinente ». On peut ainsi considérer qu'il existe un couplage fort entre un processus d'attribution de sens et l'accomplissement même de l'action dont il émerge. Même si nous mobilisons des cadres d'analyse qualitative permettant des interprétations des contextes, la signification de l'action dans ces contextes n'est pas donnée a priori mais construite par l'acteur in situ. Il est donc essentiel, dans une perspective compréhensive, de pouvoir accéder à cette signification dont seul l'acteur peut rendre compte. Or, ces significations, sont relativement transparentes pour l'acteur et donc exprimables (de façon partielle) sous certaines conditions que des méthodologies telles que l'auto confrontation par exemple (méthodologie très utilisée dans les différents dispositifs) permettent d'installer.

Cette importance accordée au point de vue subjectif de l'acteur est justifiée, selon les dispositifs, par des options théoriques diverses, renvoyant au postulat de conscience préréflexive dans le cadre des dispositifs se réclamant de l'anthropologie cognitive (Theureau, 2004, 2006), à la distinction entre conscience réfléchie et conscience directe dans la perspective psycho-phénoménologique (Vermersch, 1996, 2000) ou encore au réel de l'activité (Clot, 1999), ce dernier dépassant largement ce qui est réalisé, observable et comportant aussi les activités empêchées ou contrariées. Selon Vygotsky, en effet, le comportement est un système de réactions qui a vaincu : ce que le sujet a vécu l'a été après une lutte, un conflit entre des activités concurrentes.

Pour autant, la plupart des dispositifs privilégient une double approche extrinsèque et intrinsèque, postulant la complémentarité des informations produites par une observation armée et celles fournies par les discours des acteurs sur leurs pratiques. 


\section{L'engagement et la posture des acteurs}

21 Ces différents éléments d'analyse amènent à penser qu'il est extrêmement difficile, dans le champ de la formation, d'imaginer et de concevoir des pratiques scientifiques et des pratiques formatives totalement autonomes (Lesne, 2001). Ils posent par conséquent la délicate question des relations entre les acteurs de ces dispositifs (chercheurs et praticiens) où la perspective formative est de plus en plus souvent incorporée explicitement au projet de recherche. Si les effets induits par la participation des enseignants à des recherches empiriques (cf. le rôle des entretiens sur la pratique réflexive...) n'appellent pas de mise en question particulière de la posture de chercheur, les intentions explicites d'aide que ce dernier peut manifester nécessitent quelques développements. Selon Schwartz (1997), dans le cas où la recherche veut servir une démarche de conseil, ces relations sont fondées sur un contrat qui énonce un double questionnement: des énoncés du chercheur par les enseignants, des actions d'enseignement par les chercheurs. D'où la nécessité de l'installation d'un rapport symétrique, horizontal entre les acteurs, le chercheur ne pouvant pas occuper une position de surplomb par rapport aux enseignants.

Il s'agit par ailleurs de coordonner les objectifs de recherche avec les objectifs de formation, ce qui appelle une définition claire, précise, explicite, contractualisée des rôles de chacun des acteurs dans la coopération engagée. Or, il se peut que ces objectifs entrent en conflit et il est toujours délicat de vouloir tenir à tout prix un objectif de recherche que les praticiens considéreraient comme incompatible avec leurs visées d'apprentissage et de développement professionnel. Le chercheur, dans ce cas, est généralement amené à abandonner son objectif de recherche au profit de celui des formés s'il ne veut rendre obsolète l'activité de coopération. Lesne (2001) prend sur ce sujet une position tranchée. Pour lui, un même acteur ne peut simultanément réaliser deux rôles s'excluant l'un l'autre (chercheur et formateur), les systèmes d'activités spécifiques du chercheur et du formateur sont trop antagonistes pour pouvoir être cumulés dans un même espacetemps. Mais il considère cependant qu'il peut alternativement (selon ses compétences) remplir ces deux rôles. Nous pensons en effet que cette dissociation dans le temps et selon les situations est indispensable pour ne pas mettre le chercheur dans une posture de tension telle qu'il sera amené à devoir trancher entre une activité d'investigation et une activité de formation et/ou de co-construction avec les enseignants. Cela d'autant plus que l'activité sacrifiée ne peut être dans l'immense majorité des cas que celle de chercheur pour des raisons aisément compréhensibles : l'abandon par les enseignants de leurs visées pragmatiques se solderait immanquablement par la mort de la coopération. Pour Blanchard Laville et coll. (2007, p. 60) la question essentielle est alors celle « de la transposition des savoirs de recherche en savoirs pour la formation et en savoirs de terrain. Qui prend en charge ces différentes transpositions qui ne peuvent pas rester dans l'implicite? Il nous semble que la résolution d'une telle question passe vraisemblablement par un travail sur le lien entre chercheurs et formateurs d'enseignants. Les formateurs sont sans doute les acteurs les mieux placés du système éducatif pour relayer les savoirs de recherche, étant des sortes d'acteurs interfaces entre la communauté professionnelle et la communauté scientifique». Or, cette proposition d'introduire une médiation supplémentaire entre savoirs de recherche et savoirs de terrain, entre communauté scientifique et communauté professionnelle ne peut que rendre encore plus délicate et aléatoire la démarche de transposition. De façon 
encore plus radicale, nous pensons même que la transposition des savoirs de recherche en savoirs pour la formation puis en savoirs de terrain ne constitue pas la réponse la plus appropriée pour faciliter l'appropriation par les enseignants des savoirs issus de la recherche. Il nous semble que c'est par la confrontation directe de ces deux types de savoirs, dont les dépositaires sont les chercheurs d'un côté, les professionnels de l'autre, par les débats que celle-ci engendre, par les conflits socio-cognitifs qu'elle suscite que se produira de façon plus pertinente ce processus de reconstruction de savoirs utiles pour l'action. Le développement d'une plus grande réflexivité, de réorganisations conceptuelles finalisées par l'action, d'activités cognitives de généralisation chez les enseignants (Pastré, Mayen, Vergnaud, 2006) nous parait avoir plus de chances d'émerger de cette confrontation directe que par le biais de médiations qui risquent d'introduire une étape d'interprétation intermédiaire qui pourrait faire perdre du sens à la confrontation.

\section{Conclusion}

Nous conclurons en tirant deux conséquences principales de l'analyse relative à ces relations entre chercheur et professionnel.

- La première est que c'est à l'enseignant-chercheur de prendre en charge ce travail de mise en confrontation avec les professionnels. Cela suppose une clarification de la mission d'enseignement de l'enseignant-chercheur dans ce contexte. Il ne s'agit plus de transmettre, par le biais de cours, les principaux produits de la recherche dans son champ mais d'accepter de confronter les travaux et leurs résultats qui lui semblent les plus pertinents pour apporter un point de vue de chercheur sur les phénomènes étudiés conjointement avec les professionnels. Cela peut bien entendu consister à apporter dans les débats des résultats de recherche produits par d'autres chercheurs, mais aussi et surtout, ses propres travaux. Cet apport de ses propres recherches nous semble constituer la voie la plus prometteuse et la plus directement en rapport avec ce qui semble devoir être la définition d'un enseignant chercheur aujourd'hui dans une formation universitaire professionnalisante.

- La seconde conséquence renvoie au statut et rôle des formateurs. La remarque précédente pourrait laisser croire que le formateur n'aurait plus grande utilité dans ce type de formation. Nous ne le pensons pas. Mais cela nécessite cependant une réflexion sur ce qui fonde l'utilité et la crédibilité des formateurs non chercheurs. Si la réponse semble plus évidente (bien qu'elle nécessite d'être argumentée) concernant les formateurs dits «de terrain » (maîtres de stage, conseillers pédagogiques...) elle devient plus délicate pour des formateurs non chercheurs et n'enseignant plus dans le premier ou le second degré, au sein d'une formation professionnalisante qui revendique haut et fort sa dimension universitaire. Penser que la confrontation entre savoirs issus de la recherche et savoirs d'expérience est essentielle ne signifie pas que d'autres types de savoirs et d'autres types d'intervention ne sont pas utiles. Mais, fonder la légitimité et la spécificité des formateurs sur l'interface qu'ils auraient à jouer entre les savoirs issus de la recherche et les savoirs de terrain ne va plus forcément de soi au regard de ce que nous disions plus haut. Des travaux de recherche nous sembleraient pouvoir être engagés sur cette question et contribuer à éclairer la question des compétences et de l'avenir de ces formateurs. 
Le petit français illustré, Journal des écoliers et des écolières. $N^{\circ} 265,1894$, Editeurs A. Colin et Cie : Paris. Source : CEDRHE.

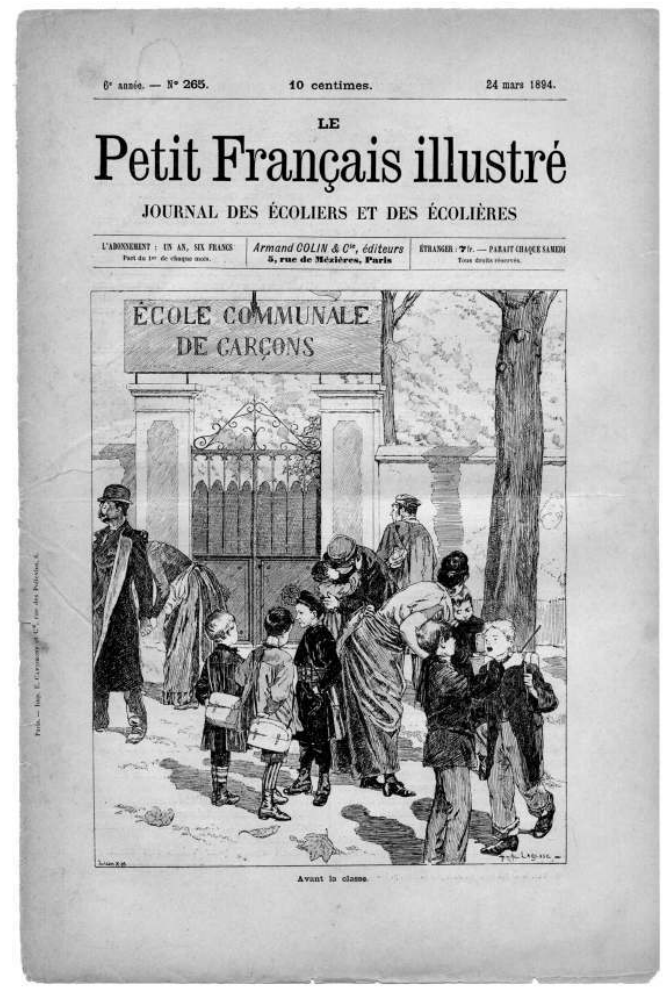

\section{BIBLIOGRAPHIE}

Amigues, R., Faïta, D. et Saujat, F., Travail enseignant et apprentissages scolaires. In Gentaz, E. et Dessus, P. Comprendre les apprentissages : Psychologie cognitive et éducation. Paris : Dunod, 2004.

Amigues, R., Faïta, D. et Kherroubi, M., Métier enseignant, organisation du travail et analyse de l'activité. Skholê, nº́sécial, 2003.

Barbier, J.M. (dir.), La formation des adultes : crise et recomposition. Questions de recherche en éducation 2 - Action et Identité, Paris, INRP, 2001.

Barbier, J.M., Les voies nouvelles de la professionnalisation. In Lenoir ,Y., Bouillier-Oudot, M.H. Savoirs professionnels et curriculum de formation. Presses Universitaires de Laval, 2006.

Billiard, M., Le sommeil normal et pathologique. Troubles du sommeil et de l'éveil. Paris Milan Barcelone, Masson, 1998.

Blanchard-Laville, C. ; Chaussecourte, P. ; Roditi, E., Recherche co-disciplinaire sur les pratiques enseignantes : quels modes de coopération avec les praticiens observés ? Éducation et Francophonie , XXXV/2, 2007, p. 45-61. 
Brousseau, G., «Théorisation des phénomènes d'enseignement des mathématiques ». Thèse de doctorat d'État. Université de Bordeaux 1, 1986.

Clot, Y., La fonction psychologique du travail. Paris : PUF, 1999.

Clot, Y., FaIta, D., Genres et styles en analyse du travail. Concepts et méthodes. Travailler, $\mathrm{n}^{\circ} 4$, 2000 , p. 7-42.

Dejours, C., Travail, usure mentale. Paris : Bayard, 1993.

De Landsheere, V., L'éducation et la formation. Paris : PUF, 1992.

Dugal, J.P., Le conseil en formation initiale des enseignants. Intérêt et fonction des savoirs didactiques pour le tutorat des professeurs stagiaires en Éducation physique et sportive. Thèse de doctorat en didactique des disciplines scientifiques et technologiques. Université Toulouse III Paul Sabatier, 2003.

Durand, M., Chronomètre et survêtement : reflets de l'expérience quotidienne d'enseignants d'éducation physique. Paris : éds revue EPS, 2000.

Durand, M., Un programme de recherche technologique en formation des adultes. Une approche enactive de l'activité humaine et l'accompagnement de son apprentissage/développement. Éducation et Didactique, ${ }^{\circ} 2 / 3,2008$, p. 97 - 121.

Espinassy, L. et Saujat, F., Enseigner les Arts Plastiques en ZEP : les dessous du métier. Recherche et Formation, n44, 2004, p. 111-124.

Goigoux, R., Analyser l'activité d'enseignement de la lecture : une monographie. Revue Française de Pédagogie, $\mathrm{n}^{\circ} 138,2002$, p. 125-134.

Grison, B., Des Sciences Sociales à l'Anthropologie Cognitive. Les généalogies de la Cognition Située, @ctivités, 1(2), 2004, p. 26-34. http://www.activites.org/v1n2/grison.pdf

Grosstephan, V., Développement professionnel d'enseignants d'EPS : processus et effets dans le cadre d'un dispositif de formation continue associant praticiens et chercheur. Thèse de doctorat non publiée. Université de Reims Champagne Ardenne, France, 2010.

Le Guillant, L., Quelle psychiatrie pour notre temps. Toulouse : Erès, 1984.

Lesne, M., Science et action : quelles relations ? Essai d'analyse. In Questions de recherche en éducation 2 Action et Identité, 2001.

Marcel, J.F., La démarche de recherche-formation. Propositions pour un trait d'union entre la recherche et la formation dans le cadre de la formation continue des enseignants. Recherche et Formation, $\mathrm{n}^{\circ} 32,1999$, p. 89-100.

Mayen, P., Effets d'apprentissage dans les interactions ordinaires tuteurs-novices. Dans Actes du congrès de l'Association des enseignants-chercheurs en sciences de l'éducation (AECSE) : Bordeaux, juillet 1999. Paris : AECSE, 1999.

Mayen, P., Intégrer les savoirs à l'action. Dans Perrenoud, P. ; Altet, M., Lessard, C., et Paquay, L., Conflits de savoirs en formation des enseignants. Bruxelles : De Boeck, 2008, p. 43-58.

Mouchet, A., Caractérisation de la subjectivité dans les décisions tactiques des joueurs d'élite 1 en rugby, Thèse de Doctorat non publiée, Université Bordeaux 2, France, 2003.

Pastre, P, Mayen, P. et Vergnaud, G., La didactique professionnelle. Note de synthèse. Revue Française de Pédagogie, n¹54, 2006, p. 145-198.

Rabardel, P., Les hommes et les technologies : approche cognitive des instruments contemporains. Paris : A. Colin, 1995. 
Roger, JL., Refaire son métier. Toulouse : Erés, 2007.

Saujat, F., Ergonomie de l'activité enseignante et développement de l'expérience professionnelle : une approche clinique du travail du professeur. Thèse de Doctorat, $n^{\circ} 13 / 12$. Aix-en-Provence : Université de Provence, 2002.

Schön, D., Le praticien réflexif. Montréal : Les Éditions Logiques, 1994.

Schön, D., Le tournant réflexif. Montréal : Les Éditions Logiques, 1996.

Schurmans, M.N., L'approche compréhensive et qualitative dans la recherche en formation. Éducation Permanente, $\mathrm{n}^{\circ}$ 177, 2008, p. 91-103.

Schwartz, Y., Travail et ergologie. In Schwartz, Y. (Ed.), Reconnaissances du travail, Paris : PUF, 1997, p. 1-33.

Theureau, J., Le cours d'action. Méthode élémentaire. Toulouse : Octarès, 2004.

Theureau,, J., Le cours d'action. Méthode développée. Toulouse : Octarès, 2006.

Varela, F.J., Autonomie et connaissance. Paris : Seuil, 1989.

Vermersch, P., Pour une psychophénoménologie. GREX, n¹3, 1996, p. 01-06.

Vermersch, P., L'explicitation phénoménologique à partir du point de vue en première personne. Expliciter, $\mathrm{n}^{\circ} 36,2000$, p. 5-11.

Wenger, E., Communities of Practice: Learning, Meaning, and Identity, Cambridge University Press, 1998.

\section{NOTES}

1. / Ingénierie didactique 1 sur le schéma $n^{\circ} 1$

2. / Ingénierie didactique 2 sur le schéma $n^{\circ} 1$

3. / Durand (2008, p. 101), "l'activité des acteurs fait a priori et fondamentalement l'objet d'une «mise au travail ", qui suppose la réalisation d'un "laboratoire ». Le principe de cette mise au travail est de transformer la structuration et la signification de l'activité pour les acteurs/formés dans une orientation consensuellement définie par le formateur et les formés. À cette fin, des environnements d'apprentissage/ développement sont conçus et mis à disposition des individus et des collectifs en formation ». La double finalisation de ce programme nous semble assez caractéristique d'une tendance générale visant à questionner l'utilité sociale de la recherche pour les professionnels impliqués. Pour Durand (2008, p. 98) « ce programme se concrétise par des pratiques de conception ayant pour objet l'activité des individus et des collectifs, et relevant de problématiques de formation spécifiques que nous considérons comme aussi cruciales au plan épistémologique et aussi utiles au plan social - et donc d'égale dignité - que les pratiques de recherche scientifique ». L'extension technologique du programme de recherche auquel il est articulé de façon organique constitue ainsi un élément essentiel de réponse à ce problème de fond. 


\section{RÉSUMÉS}

Cet article propose une réflexion critique sur les dispositifs organisant la rencontre entre chercheurs et professionnels de l'enseignement. Il interroge notamment, à partir de l'étude de neuf types de dispositifs, les finalités qui leur sont fixées. L'analyse montre notamment que l'engagement et la posture adoptés par les acteurs dans ces dispositifs constituent une variable fondamentale de la réussite de cette rencontre.

This article submits a critical reflection about the devices organizing the meeting of researchers and teaching professionals. In particular, it considers, from the study of nine kinds of devices, their aims. The analysis particularly points out that the commitment and the position adopted by the actors in those devices form an essential variable for the success of this meeting.

INDEX

Keywords : devices., research, teaching, Training

Mots-clés : dispositifs, enseignement, Formation, recherche

\section{AUTEUR}

\section{VINCENT GROSSTEPHAN}

MCF 70e, CEREP (EA 4672), IUFM Champagne Ardenne,Université de Reims Champagne Ardenne 